\title{
Error Bounded Padé Approximation via Bilinear Conformal Transformation
}

\author{
Chung-Ping Chen ${ }^{1}$ and D. F. Wong ${ }^{2}$ \\ ${ }^{1}$ Strategic CAD Labs, Intel Corp., Hillsboro, Oregon 97124. \\ ${ }^{2}$ Department of Computer Sciences, University of Texas at Austin, Austin, Texas 78712.
}

\begin{abstract}
Since Asymptotic Waveform Evaluation (AWE) was introduced in [5], many interconnect model order reduction methods via Padé approximation have been proposed. Although the stability and precision of model reduction methods have been greatly improved, the following important question has not been answered: "What is the error bound in the time domain?". This problem is mainly caused by the "gap" between the frequency domain and the time domain, i.e. a good approximated transfer function in the frequency domain may not be a good approximation in the time domain. All of the existing methods approximate the transfer function directly in the frequency domain and hence can not provide error bounds in the time domain. In this paper, we present new moment matching methods which can provide guaranteed error bounds in the time domain. Our methods are based on the classic work by Teasdale in [1] which performs Padé approximation in a transformed domain by the bilinear conformal transformation $s=\frac{1-z}{1+z}$.
\end{abstract}

\section{Introduction}

In the last few years, the AWE algorithm based on Padé approximation has emerged as an efficient method to analyze large linear circuits. However, AWE suffers from numerical instability which makes this method hard to obtain more than a few accurate poles. For this reason, many new algorithms such as CFH [6], Lanczos process [3, 13, 14], Arnoldi process [4, 12], Congruence Transformation [8, 9], and Balance Truncation [10] have been proposed.

Although the numerical stability of model order reduction methods has been significantly improved, there are no guaranteed error bounds in the time domain for these methods due to the fact that they only approxi- mate the transfer function in the frequency domain.

In [1], Teasdale introduces a different approximation method which performs Padé approximation in a transformed domain by the bilinear conformal transformation $s=\frac{1-z}{1+z}$. Teasdale then shows that this approach not only has an error bound in the time domain but also minimizes the least square error of this bound. On the other hand, this approach assumes that the analytical transfer function is known a priori which unfortunately limits its practical applicability.

In this paper, we provide practical implementations of [1] by computing the Taylor expansion of the transfer function in the bilinear transformed domain. Our algorithms, without the knowledge of the analytical expression of the transfer function, utilize a moment computation procedure similar to the AWE method to perform Padé approximation in the transformed domain. With minor modification of the approximation procedure, our methods can also be embedded into the Lanczos/Arnoldi type algorithms to improve numerical stability.

The organization of the remainder of this paper is as follows. In section 2, we briefly review the background of linear circuit simulation, Padé approximation, AWE, PVL (Padé via Lanczos process), and Arnoldi process. In section 3, we introduce the new Padé approximation via bilinear conformal transformation and its practical implementations. In the last section, we show some experimental results.

\section{Preliminaries}

By the modified nodal analysis, the systems equations of linear circuits can be expressed as follows

$$
C \dot{x}=-G x+b u,
$$

where $x$ represents the state variables, $G$ and $C$ represent the conductance and susceptance matrices, and the 
term $b u$ represents excitation from independent sources. Applying Laplace transform to Equation (1) and assuming zero initial conditions (i.e. $x(0)=0$ ), we get

$$
s C X(s)=-G X+b U(s),
$$

where $X(s)$ and $U(s)$ denote the Laplace transform of $x$ and $u$, respectively. After rearranging the terms of the above equation, we get the impulse response of the system as follows

$$
X(s)=(s C+G)^{-1} b .
$$

Let $A=-G^{-1} C$, we can rewrite the above equation as

$$
X(s)=(I-s A)^{-1} G^{-1} b .
$$

Let $v=G^{-1} b$. The AWE method expands the above equation at $s=0$ or $s=\infty$ and get

$$
X(s)=\sum_{i=0}^{\infty} A^{i} v s^{i} .
$$

Let $m_{i}=A^{i} v$. The AWE method uses the following recurrent relation to iteratively compute higher order moments from lower order moments.

$$
\begin{aligned}
m_{0} & =v=G^{-1} b \\
m_{i} & =A m_{i-1} .
\end{aligned}
$$

Note that it only needs to perform $L U$ decomposition of $A$ once. The rest of the computation is on repeatedly solving the above linear equations. To get more insight of the AWE method, we have the following observations. By the definition of $A$, we know

$$
\begin{aligned}
G m_{0} & =b \\
G m_{i}+C m_{i-1} & =0, \quad i \geq 1 .
\end{aligned}
$$

Hence, to calculate $m_{q}$, we can first substitute each capacitor and inductor with a current and voltage source, respectively, with the value of $\mathrm{Cm}_{q-1}$ and then solve for voltages and currents of the original circuit while keeping resistors and conductors unchanged. The transformations are summarized in Figure 1. However, this type of methods to compute moments are equivalent to the classic inversion power iteration method in [7] to calculate eigenvalues and eigenvectors. It is well known that this type of methods has difficulty to get more than a few dominant eigenvalues. Let $v_{i}=A^{i} v$. As $i$ innreases, $v_{i}$ is getting more linear dependent with the
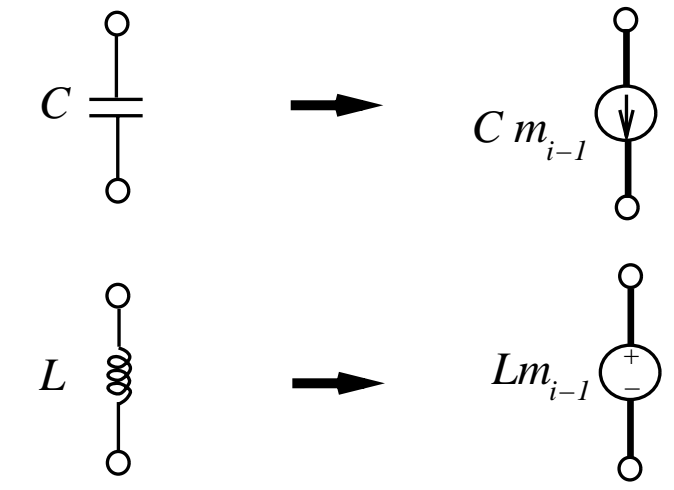

Figure 1: The transformations of the AWE method.

Krylov space which is defined as $\operatorname{span}\left\{v, A^{1} v, A^{2} v, \ldots\right\}$. Although several remedies such as frequency shifting and moment shifting have been proposed, the nature of this difficulty remains.

Recently, [3, 4] propose several Lanczos/Arnoldi type algorithms [7] to calculate the Padé approximations which have better numerical stability. Instead of using information only from the last iteration, these methods utilize the information from multiple previous iterations. All of these iterative methods are based on the following equation:

$$
A V_{q}=V_{q} H_{q}+h_{q+1, q} v_{q+1} e_{q}^{T},
$$

where $e_{q}$ is the $q^{\text {th }}$ unit vector in $\mathbf{R}^{q}, V_{q}$ is the set of the Lanczos vectors $\left\{v_{1}, v_{2}, \ldots, v_{q}\right\}$, and $H_{q}=\left(h_{i, j}\right)_{q \times q}$ is an $q$-th order approximation matrix of $A$. $H_{q}$ is a Heisenberg matrix for the Arnoldi algorithm and is a tridiagonal matrix for the Lanczos algorithm. The Lanczos vector $V_{q}$ satisfies

$$
V_{q} V_{q}^{T}=I .
$$

Equation (4) suggests the following approximation of $A$

$$
A=V_{q} H_{q} V_{q}^{T} .
$$

By substituting (6) back into (3), we get

$$
\begin{aligned}
X(s) & =\left(I-s V_{q} H_{q} V_{q}^{T}\right)^{-1} v \\
& =V_{q}\left(I-s H_{q}\right)^{-1} V_{q}^{T} v .
\end{aligned}
$$

Let $v_{1}=\frac{v}{\|v\|}$. By (5), we know $V_{q}^{T} v=\|v\| e_{1}$. By Equation (7), we know

$$
X(s)=\|v\| V_{q}\left(I-s H_{q}\right)^{-1} e_{1} .
$$


Again, we expand $X(s)$ at $s=0$ and get

$$
X(s)=\|v\| \sum_{i=0}^{\infty} V_{q} H_{q}^{i} e_{1} s^{i} .
$$

By the above equation, we can compute the new moments as follows

$$
m_{i}=\|v\| V_{q} H_{q}^{i} e_{1} .
$$

It can be shown that under a mild assumption, the eigenvalues of $H_{q}$ reasonably approximate dominant eigenvalues of $A$ [7]. [3] further gave error bounds for the approximated poles.

There are several other variations of Lanczos/Arnoldi type model reduction algorithms. [8,9] uses the properties of congruence transformation to guarantee the passivity of the reduced system. [10] takes advantage of the properties of singular values to bound the errors of the transfer function in the frequency domain. [11] extends model order reduction methods to deal with several transmission line issues.

\section{PVBCT Algorithm and Practical Im- plementations}

Although Lanczos/Arnoldi process gives error bounds of the poles of the transfer function, the error bounds in the time domain are not known. [1] introduces a method which performs Padé approximation in a bilinear transformed domain and then shows an error bound in the time domain. It then proves that this method actually minimizes the least square error of this error bound. We call this algorithm PVBCT (Padé approximation Via Bilinear Conformal Transformation). The major disadvantage of PVBCT is that it assumes the transfer functions are known a priori, thus a practical implementation may not be possible. In this section, we first introduce the PVBCT method and then give two practical implementations of the algorithm.

\subsection{PVBCT Algorithm}

Given a transfer function $X(s)$, [1] first applies the bilinear conformal transformation $s=\frac{1-z}{1+z}$ to $X(s)$ and gets $X^{\prime}(z)=X\left(\frac{1-z}{1+z}\right)$. It removes the zeros of $X^{\prime}(z)$ at $z=-1$ by multiplying $(1+z)^{-N}$ to $X^{\prime}(z)$, where $N$ is the number of zeros of $X^{\prime}(z)$ when $z=-1$. It then performs the Padé approximation to $X^{\prime}(z)$ in the $z$ domain. This function is transformed back to the $s$ domain under the reverse transformation $z=\frac{1-s}{1+s}$.
Finally, it shifts the DC value to match the final DC value.

[1] gives the following theorem about the error bound of PVBCT in the time domain.

Theorem 1 Given a transfer function $X(s)$, the error $\epsilon(t)=|x(t)-\widehat{x}(t)|$ between the original transient response $x(t)$, and the approximated transfer function $\widehat{X}(s)$ in the time domain of PVBCT, satisfies the following inequality

$$
|\epsilon(t)|^{2} \leq \frac{2^{2 N-3} \pi^{2} \Gamma\left(N-\frac{3}{2}\right)}{3 \Gamma(N-1)} \int_{-\pi}^{\pi}\left|X_{1}\left(e^{j \theta}\right)-\widehat{X_{1}}\left(e^{j \theta}\right)\right|^{2} d \theta,
$$

where $N$ is the number of zeros of $X^{\prime}(z)$ when $z=-1$.

The following theorem from [1] also shows that this method minimizes the least square error in the time domain.

Theorem 2 Given a transfer function $X(s)$, the approximated transfer function $\widehat{X}(s)$ minimizes the least square error in the time domain.

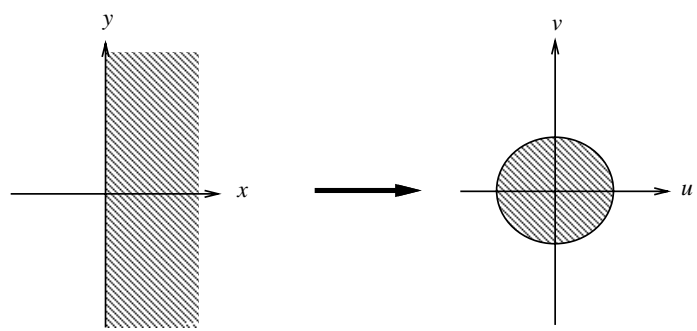

Figure 2: The conformal transformation $\frac{1-z}{1+z}$.

PVBCT can potentially get more useful poles than the AWE method. According to CFH [6], the most influential poles for the transient response are located near the $j \omega$ axis. Hence CFH computes the Lorents series at multiple points in the $j \omega$ axis. As shown in Figure 2, this bilinear conformal transformation $s=\frac{1-z}{1+z}$ maps the right halfplane of $s$ domain onto the interior of the unit circle centered in the origin of the $z$ domain, the $j \omega$ axis onto the unit circle. Hence the poles near the $j \omega$ axis in the $s$ domain will be relocated near the unit circle around the original points in the $z$ domain.

The major difficulty of this algorithm is that it as-umes the analytical form of the transfer function is known a priori. In the next two subsections, we present two practical implementations of PBVCT via Lanczos/Arnoldi and Inversion Power Iteration method, re- 
-pectively. These algorithms can obtain the approximated transfer function in the transformed domain without the knowledge of the analytical form of $X(s)$.

\subsection{PVBCT via Lanczos/Arnoldi process}

In this subsection, we present the practical implementation of PVBCT via Lanczos/Arnoldi process.

From Equation (2), we know

$$
\frac{1-z}{1+z} C X(z)=-G X(z)+b U(z) .
$$

Multiplying both sides with $(1+z)$, we get

$$
(1-z) C X(z)=-(1+z) G X(z)+(1+z) b U(z) .
$$

The above equation can be rewritten as

$$
X(z)=((C+G)+z(G-C))^{-1}(1+z) b U(z)
$$

Let $A=(C+G)^{-1}(C-G)$ and $v=(C+G)^{-1} b U(z)$. We have

$$
X(z)=(I-z A)^{-1} v(1+z) .
$$

The Taylor expansion of $X(z)$ at $z=0$ is

$$
X(z)=\sum_{i=0}^{\infty} A^{i} v z^{i}(1+z) .
$$

The moments of the impulse response $(U(z)=1)$ are given by

$$
\begin{aligned}
& m_{0}=v \\
& m_{i}=\left(A^{i}+A^{i-1}\right) v, \quad i \geq 1 .
\end{aligned}
$$

After performing $q$ steps of Lanczos/Arnoldi approximation, we get

$$
A=V_{q} H_{q} V_{q}^{T} .
$$

By substituting (10) back to (9), we get

$$
\begin{aligned}
X(z) & =\left(I-z V_{q} H_{q} V_{q}^{T}\right)^{-1} v(1+z) \\
& =V_{q}\left(I-z H_{q}\right)^{-1} V_{q}^{T} v(1+z) .
\end{aligned}
$$

Let $v_{1}=\frac{v}{\|v\|}$. Hence, by (5), we know $V_{q}^{T} v=\|v\| e_{1}$. Hence, by Equation (11), we know

$$
X(z)=\|v\| V_{q}\left(I-z H_{q}\right)^{-1}(1+z) e_{1} .
$$

The Taylor expansion of $X(z)$ is

$$
X(z)=\|v\| \sum_{i=0}^{\infty} V_{q} H_{q}^{i} e_{1} z^{i}(1+z) .
$$

The moments in the $z$ domain can be computed by

$$
\begin{aligned}
& m_{0}=v \\
& m_{i}=\|v\| V_{q}\left(H_{q}^{i}+H_{q}^{i-1}\right) v, \quad i \geq 1 .
\end{aligned}
$$

According to the above discussions, we derived a practical implementation of PVBCT based on the Lanczos/Arnoldi method. The new algorithm is summarized in Figure 3.
Algorithm: PVBCT-LA (Padé approximation via bilinear conformal transformation by Lanczos/Arnoldi process) Input: A circuit with $G, C, b$ matrices. Output: An approximated transfer function $\widehat{X}(s)$

A1 Let $A=(C+G)^{-1}(C-G)$ and $v=(C+G)^{-1} b$

A2 Apply $q$ steps of Lanczos/Arnoldi algorithm to $A$ and get $V_{q}, H_{q}$.

A3. Let $\widehat{X}(z)=\|v\| V_{q}\left(I-z H_{q}\right)^{-1}(1+z) e_{1}$.

A4. Remove all the zeros of $\widehat{X}(z)$ at $z=-1$ by forming $\widehat{X_{1}}(z)=\frac{X(z)}{(1+z)^{N}}$ Twhere $N$ is the number of zeros of $\widehat{X}(-1)$.

A5. Perform Padé approximation on $\widehat{X_{1}}(z)$ and get $\widehat{X_{2}}(z)$.

A6. Get the new approximation by $\widehat{X}(s)=K \widehat{X_{2}}\left(\frac{1-s}{1+s}\right) \Gamma$ where $K$ is a constant which makes $X(0)=\widehat{X}(0)$.

Figure 3: Padé approximation via bilinear conformal transformation by Lanczos/Arnoldi process.

\subsection{PVBCT via Inverse Power Iteration}

In this subsection, we show how to implement PVBCT by the Inverse Power Iteration method.

Let $\frac{1-z}{1+z}=\sum_{i=0}^{\infty} a_{i} z^{i},(9)$ can be rewritten as

$$
C \sum_{i=0}^{\infty} a_{i} z^{i} X(z)=-G X(z)+b U(z) .
$$

Let $X(z)=\sum_{i=0}^{\infty} x_{i} z^{i}$ and $b U(z)=\sum_{i=0}^{\infty} b_{i} z^{i}$, we can rewrite the above equation as

$$
C \sum_{i=0}^{\infty}\left(\sum_{j=0}^{i} a_{j} x_{i-j}\right) z^{i}=-G \sum_{i=0}^{\infty} x_{i} z^{i}+\sum_{i=0}^{\infty} b_{i} z^{i} .
$$

Combining the coefficient of $z^{i}$, we get

$$
\sum_{i=0}^{\infty}\left(G x_{i}+C \sum_{j=0}^{i} a_{j} x_{i-j}\right) z^{i}=\sum_{i=0}^{\infty} b_{i} z^{i}
$$

By the above equation, the moments $\left\{x_{0}, x_{1}, \ldots\right\}$ of $X(z)$ satisfy

$$
\begin{aligned}
G x_{0}+a_{0} C x_{0} & =b_{0} \\
G x_{i}+C a_{0} x_{i}+C \sum_{j=0}^{i-1} a_{j} x_{i-j} & =\quad b_{i}, \quad i \geq 1 .
\end{aligned}
$$


The above equations give an iterative method to compute the moments. It shows that to compute the moments of the capacitors we only have to transform the capacitor to an independent current source connected in parallel to a voltage control current source and solving for the voltage of this device as shown in Figure 4. For a capacitor with value $C$, the value of the independent current source is $C \sum_{j=0}^{i-1} a_{j} x_{i-j}$ and the value of the voltage control current source is $a_{0} * C * \operatorname{Voltage}(C)$, where Voltage $(C)$ is the resultant voltage difference between the two end of the device. The new moment of this capacitor will be $x_{i}=\operatorname{Voltage}(C)$. For inductors, we transform each inductor to an independent voltage source serially connected to a current control voltage source as shown in Figure 4. For an inductor with value $L$, the value of the independent voltage source is $L \sum_{j=0}^{i-1} a_{j} x_{i-j}$ and the value of the voltage control current source is equal to $a_{0} * L * \operatorname{Current}(L)$, where Current $(L)$ is the resultant current flowing through the device. The new moment of this inductor will be $x_{i}=$ Current $(L)$.

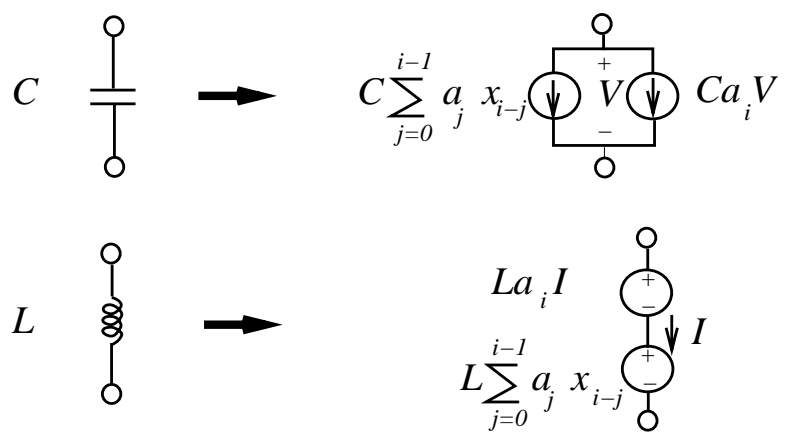

Figure 4: The transformations of PVBCT.

According to the above discussion, we have derived a practical implementation of PVBCT based on the Inverse Power Iteration method. This method is summarized in Figure 5.

\section{Experimental Results and Concluding Remarks}

We have implemented and tested our algorithms on a PC with Pentium-133 microprocessor using many clock tree examples [2]. In Figure 6, we show the comparison between PVBCT-IPI, AWE, and SPICE output when the modeling of the clock trees contain RLC elements. An enlarged plot of the beginning part of Figure 6 is shown in Figure 7. It shows that the results of PVBCT-
Algorithm: PVBCT-IPI (Padé approximation via bilinear conformal transformation by Inverse Power Iteration.) Input: A circuit with $G, C, b$ matrices.

Output: An approximated transfer function $\widehat{X}(s)$.

A1 Use the simple transformation shown in Figure 4.

A2 Solve the voltages and currents of the capacitors and inductors.

A3. Compute higher order moments base on Equation (12).

A4. Repeat A1 to A3 $q$ steps.

A4. Let $\widehat{X_{0}}(z)=\sum_{i=0}^{q} m_{i} z^{i}$

A2. Remove all the zeros of $\widehat{X}(z)$ at $z=-1$ by forming $\widehat{X_{1}}(z)=\frac{\widehat{X_{0}}(z)}{(1+z)^{N}} \Gamma$ where $N$ is the number of zeros of $\widehat{X}(-1)$.

A3. Perform Padé approximation on $\widehat{X_{1}}(z)$ and get $\widehat{X_{2}}(z)$.

A4. Get the new approximation by $\widehat{X}(s)=K \widehat{X_{2}}\left(\frac{1-s}{1+s}\right) \Gamma$ where $K$ is a constant which makes $X(0)=\widehat{X}(0)$.

Figure 5: Padé approximation via bilinear conformal transformation by Inverse Power Iteration.

IPI have better fits than AWE. The simulation results for pure $\mathrm{RC}$ elements are shown in Figure 8. In this case, the transient responses from SPICE and PVBCTIPI are identical.

On the other hand, we also observe that the behavior of PVBCT-IPI is not as stable as the AWE method. Also, similar to AWE, it is not easy to find a correct order to get a good approximation. Nevertheless, we believe that it is very important to have an efficient interconnect simulation method which can provide error bounds in the time domain.

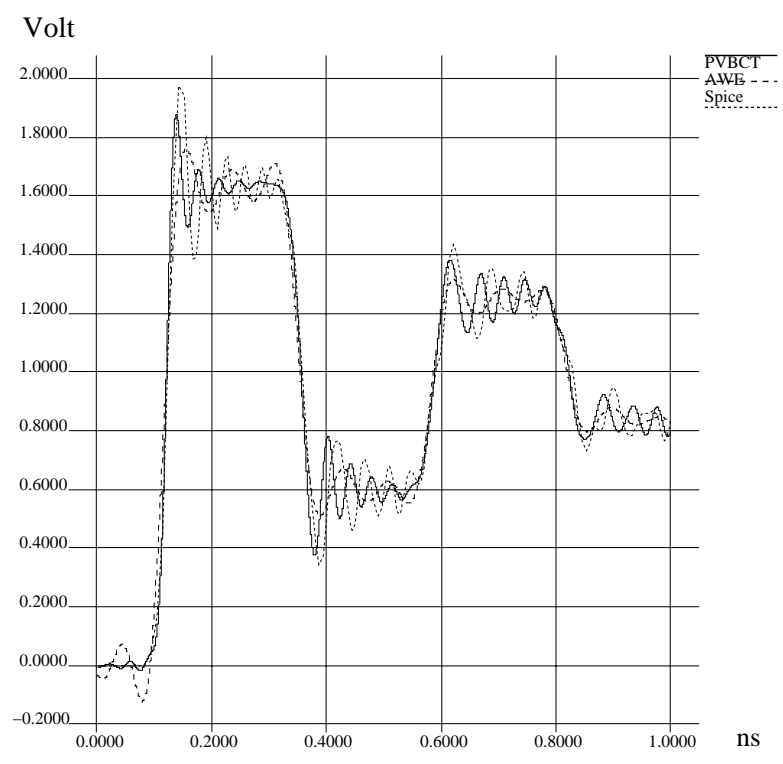

Figure 6: RLC circuit simulation result. 


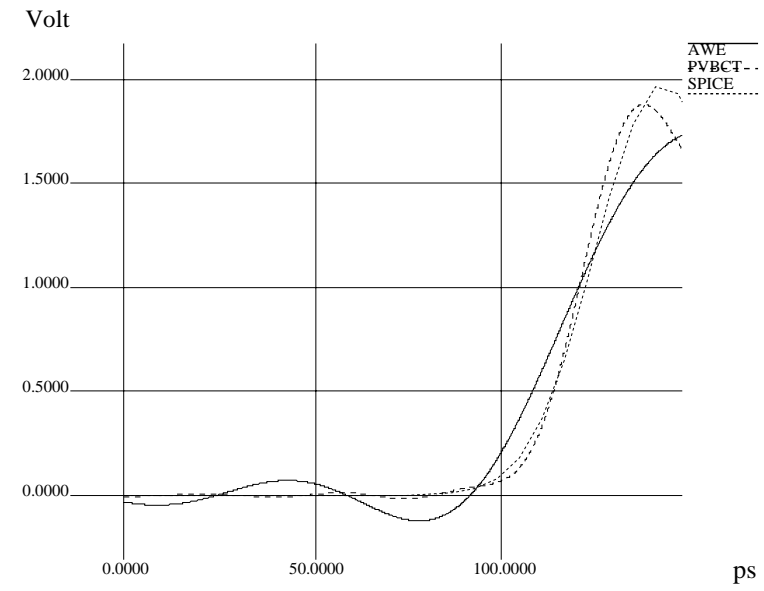

Figure 7: Enlarged RLC circuit simulation result.

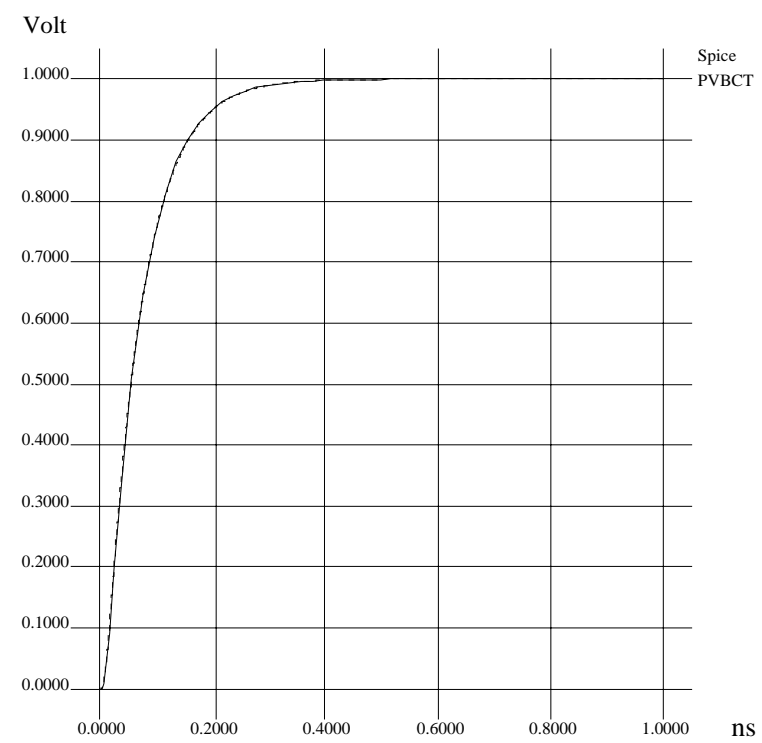

Figure 8: RC circuit simulation result.

\section{References}

[1] R.D. Teasdale, "Time domain approximation by use of Padé approximants", IRE Convention Record, Vol.1, pt.5 pp. 89-94, 1953.

[2] R. S. Tsay, "An exact zero-skew clock routing algorithm" IEEE Transactions on Computer-Aided Design of Integrated Circuits and Systems, February 1993.

[3] P. Feldmann, R. W. Freund, "Efficient linear circuit analysis by Padé approximation via the Lanczos process", IEEE Transactions on Computer-Aided Design of Integrated Circuits and Systems, May 1995.
[4] L. M. Silveira, M. Kamon, I. Elfadel, and J. White, "A coordinate-transformed Arnoldi algorithm for generating guaranteed stable reduced-order models of RLC circuits" Proc. ICCAD, 1996.

[5] L. T. Pillege, and R. A. Rohrer "Asymptotic waveform evaluation for timing analysis", IEEE Transactions on Computer-Aided Design of Integrated Circuits and Systems, April, 1990.

[6] E. Chiprout, and M. S. Nakhla, "Analysis of interconnect networks using complex frequency hopping", IEEE Transactions on Computer-Aided Design of Integrated Circuits and Systems, February, 1995.

[7] G. H. Golub and C. F. Van Loan, "Matrix computations", The Johns Hopkins University Press, Baltimore, Maryland, 1983.

[8] K.J. Kerns, I.L. Wemple, and A.T. Yang, "Stable and efficient reduction of large, multiport RC networks by pole analysis via congruence transformations", 33th ACM/IEEE DAC, 1996.

[9] A. Odabasioglu, M. Celik, and L. Pilleggi, "PRIMA: Passive reduced-order interconnect macromodeling algorithm", Proc. ICCAD, 1998.

[10] P. Rabiei, and M. Pedram, "Model Order Reduction for Large Circuit Using Balance Truncation", Proc. ASP-DAC, 1999.

[11] Qingjian Yu, Janet M. Wang, and Ernest S. Kuh, "Multipoint Moment Matching Model For Multiport Distributed Interconnect Networks", Proc. ICCAD, 1998.

[12] I.M. Elfadel, and D.D. Ling, "A Block Rational Arnoldi Algorithm for Multipoint Pawwive ModelOrder Reduction of Multiport RLC Networks", Proc. ICCAD, 1997.

[13] T.V. Nguyen, and J. Li, "Multipoint Padé Approximation Using a Rational Block Lanczos Algorithm", Proc. ICCAD, 1997.

[14] K. Gallivan, E. Grimme, and P. Wan Dooren, "Asymptotic waveform evaluation via a Lanczos method", App. Math. Lett., vol. 7, pp. 75-80, 1994. 